See discussions, stats, and author profiles for this publication at: https://www.researchgate.net/publication/257858409

\title{
On the Oscillation of Eigenfunctions of a Fourth-Order Spectral Problem
}

Article in Doklady Mathematics · May 2012

DOl: 10.1134/S1064562412030155

CITATIONS

3 authors, including:

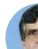

Nazim B. Kerimov

Mersin University,Mersin, Turkey

48 PUBLICATIONS 513 CITATIONS

SEE PROFILE

Some of the authors of this publication are also working on these related projects:
READS

65

Ziyatkhan S. Aliev

Baku State University

59 publications 321 Citations

SEE PROFILE

Sturm-Liouville View project 


\title{
On the Oscillation of Eigenfunctions of a Fourth-Order Spectral Problem
}

\author{
N. B. Kerimov ${ }^{a}$, Z. S. Aliev ${ }^{b}$, and E. A. Agaev $^{b}$ \\ Presented by Academician V.A. Il'in January 4, 2012
}

Received December 28, 2011

DOI: $10.1134 / \mathrm{S} 1064562412030155$

Consider the spectral problem for the fourth-order ordinary differential equation

$$
\begin{aligned}
\ell(y)(x) \equiv & \left(p(x) y^{\prime \prime}(x)\right)^{\prime \prime}-\left(q(x) y^{\prime}(x)\right)^{\prime}+r(x) y(x) \\
& =\lambda \tau(x) y(x), \quad 0<x<l,
\end{aligned}
$$

with boundary conditions

$$
\begin{gathered}
\left(p y^{\prime \prime}\right)(0)-C_{0} T y(0)-C_{1} y^{\prime}(0)=0, \\
y(0)+C_{2} T y(0)-C_{0} y^{\prime}(0)=0, \\
\left(p y^{\prime \prime}\right)(l)+D_{0} T y(l)+D_{1} y^{\prime}(l)=0, \\
y(l)-D_{2} T y(l)+D_{0} y^{\prime}(l)=0,
\end{gathered}
$$

where $\lambda \in \mathbf{C}$ is a spectral parameter, $T y \equiv\left(p y^{\prime \prime}\right)^{\prime}-q y^{\prime}$, the functions $p(x), r(x)$ are strictly positive and continuous on $[0, l]$, the function $p(x)$ has absolutely continuous derivative on $[0, l]$, the function $q(x)$ is nonnegative and absolutely continuous on $[0, l]$, and $C_{i}, D_{i} \geq 0$ with $i=0,1,2$ are real constants.

The real case of problem (1), (2) with $q(x) \equiv 0$ was studied in [1], where the class of regular and completely regular Sturmian systems was introduced and studied. For completely regular Sturmian systems, it was proved that the eigenvalues of these problems are real and form an infinite increasing sequence; moreover, in the case $r(x) \equiv 0$, all of them are positive and simple, and the corresponding eigenfunctions have Sturm oscillation properties (i.e., the number of zeros of each eigenfunction equals the number of the eigenvalue minus one), and in the case $r(x) \neq 0, x \in[0, l]$, there exists an $m_{0} \in N \cup 0$ such that all eigenvalues of the problems, except, possibly, the first $m_{0}$ of them, are simple, and the corresponding eigenfunctions with

\footnotetext{
${ }^{a}$ Mersin University, Mersin, Turkey

${ }^{b}$ Department of Mathematical Analysis, Baku State University, ul. Akademika Zakhid Khalilova 23, Baku, AZ 1148, Azerbaijan

e-mail:z_aliyev@mail.ru
}

number greater than $m_{0}$ have Sturm oscillation properties.

The structure of root subspaces and the number of zeros in $(0, l)$ of the eigenfunctions corresponding to eigenvalues with numbers at most $m_{0}$. have not been studied so far. This paper is devoted to these questions.

Let $E$ denote the Banach space $C^{3}[0, l] \cap B . C$. endowed with the norm $\|y\|_{3}=\sum_{j=0}^{3}\left|y^{(j)}\right|_{0}$, where B.C. is the set of functions satisfying boundary conditions (2) and $|\cdot|_{0}=\max _{x \in[0, l]}|y(x)|$.

By $S_{k}^{v}$, where $k \in N$, and $v=+$ or - , we denote the set of functions $y(x) \in E$ satisfying the following conditions:

(i) $y(x)$ has precisely $k-1$ zeros in the interval $(0, l)$;

(ii) $\lim _{x \rightarrow 0} v \operatorname{sign} y(x)=1$;

(iii) if $y(\xi)=0$ or $y^{\prime \prime}(\xi)=0$, then $y^{\prime}(x) T y(x)<0$ in some neighborhood of $\xi \in(0, l)$, and if $y^{\prime}(\eta)=0$ or $T y(\eta)=0$ then $y(x) y^{\prime \prime}(x)<0$ in some neighborhood of $\eta \in(0, l)$.

The sets $S_{k}=S_{k}^{+} \cup S_{k}^{-}$with $k \in N, v=+$ or - , are open subsets in $E$ [2].

Note that if $h(x), x \in[0, l]$, is a solution of the second-order initial value problem

$$
\begin{aligned}
& -\left(p u^{\prime}\right)^{\prime}+q u=0, \\
& u(l)=1, u^{\prime}(l)=0,
\end{aligned}
$$

then $h(x)>0$ for $x \in[0, l]$ and $h^{\prime}(x)<0$ for $x \in[0, l)$.

The change of variable

$$
t=t(x)=l \omega^{-1} \int_{0}^{x} h(s) d s,
$$


where $\omega=\int_{0}^{l} h(s) d s$, transforms each function $y(x) \in C^{3}[0, l]$ into a function $y(t) \in C^{3}[0, l]$ of the form

$$
\begin{gathered}
y(t)=y(x(t)), \quad \dot{y}(t)=l^{-1} \omega h^{-1}(x(t)) y^{\prime}(x(t)), \\
\ddot{y}(t)=l^{-2} \omega^{2} h^{-2}(x(t))\left\{y^{\prime \prime}(x(t))\right. \\
\left.-h^{-1}(x(t)) h^{\prime}(x(t)) y^{\prime}(x(t))\right\},
\end{gathered}
$$

$\left.(\tilde{p}(t) \ddot{y}(t))^{\prime \prime}=\left(p(x(t)) y^{\prime \prime}(x(t))\right)\right)^{\prime \prime}-q(x(t)) y^{\prime}(x(t)), t \in[0, l]$, where $\tilde{p}(t)=\left(l \omega^{-1} h(x(t))\right)^{3} p(x(t))[3]$.

Under the change of variable (5), Eq. (1) transforms into the equation

$$
(\tilde{p}(t) \ddot{y}(t))^{*}+\tilde{r}(t) y(t)=\lambda \tilde{\tau}(t) y(t), 0<t<l
$$

and the boundary conditions (2), into the boundary conditions

$$
\begin{gathered}
\tilde{p}(0) \ddot{y}(0)-\tilde{C}_{0} \tilde{T} y(0)-\tilde{C}_{1} \dot{y}(0)=0, \\
y(0)+C_{2} \tilde{T} y(0)-\tilde{C}_{0} \dot{y}(0)=0, \\
\tilde{p}(l) \ddot{y}(l)+\tilde{D}_{0} T y(l)+\tilde{D}_{1} \dot{y}(l)=0, \\
y(l)-D_{2} \tilde{T} y(l)+\tilde{D}_{0} \dot{y}(l)=0,
\end{gathered}
$$

where

$$
\begin{gathered}
r(t)=r(x(t)), \quad \tilde{\tau}(t)=l^{-1} \omega h^{-1}(x(t)) r(x(t)), \\
\tilde{T} y(t)=(\tilde{p}(t) \ddot{y}(t))=T y(x(t)), \\
\tilde{C}_{0}=l \omega^{-1} h(0), \\
\tilde{C}_{1}=l^{2} \omega^{-2} h^{2}(0)\left\{C_{1}-h^{-1}(0) h^{\prime}(0)\right\}, \\
\tilde{D}_{0}=l \omega^{-1} D_{0}, \quad \tilde{D}_{1}=l^{2} \omega^{-2} D_{1} .
\end{gathered}
$$

Problem (6), (7) is a completely regular Sturmian system (see [1]). We set

$$
\begin{aligned}
\tilde{r}_{0}=\min _{t \in[0, l]} \tilde{r}(t), & \tilde{r}_{1}=\max _{t \in[0, l]} \tilde{r}(t), \\
\tilde{\tau}_{0}=\min _{t \in[0, l]} \tilde{\tau}(t), & \tilde{\tau}_{1}=\max _{t \in[0, l]} \tilde{\tau}(t),
\end{aligned}
$$

and let (A) denote the completely regular Sturmian system is obtained from (6), (7) by replacing $\tilde{r}(t)$ by $\tilde{r}_{0}$ and $\tilde{\tau}(t)$ by $\tilde{\tau}_{1}$. Under the change $\lambda^{\prime}=\lambda \tilde{\tau}_{1}-\tilde{r}_{0}$, system (A) transforms into an equivalent system (B). Let $\lambda_{n}^{\prime}$, $n \in N$, be the $n$th eigenvalue of system (B), and let $\lambda_{n}^{(0)}=\frac{\lambda_{n}^{\prime}+\tilde{r}_{0}}{\tilde{\tau}_{1}}, n \in N$, be the $n$th eigenvalue of system (A).

Consider the nonnegative integer $m_{0}$ determined by the relations

$$
\begin{gathered}
\lambda_{m_{0}+1}^{\prime}>\frac{\tilde{\tau}_{1} \tilde{r}_{1}-\tilde{\tau}_{0} \tilde{r}_{0}}{\tilde{\tau}_{0}} \geq \lambda_{m_{0}}^{\prime} \text { and } \lambda_{m_{0}+1}^{(0)}>0, \\
\text { where } \lambda_{0}^{\prime}=-\infty .
\end{gathered}
$$

By virtue of Lemma 3 and the remark in [1, Section 13], the eigenvalues $\lambda_{1}, \lambda_{2}, \ldots, \lambda_{m_{0}}, \lambda_{m_{0}+1}, \ldots$ of the completely regular systems (6), (7) and (1), (2) except, possibly, the first $m_{0}$ of them are positive and simple, and the eigenfunction $y_{k}(x)$ corresponding to an eigenvalue $\lambda_{k}, k>m_{0}$ has $k-1$ simple zeros in the interval $(0, l)$. Then, by virtue of Lemma 1.2 from [4], we have $y_{k} \in S_{k}^{v}$, for $k=m_{0}+1, m_{0}+2, \ldots$ These assertions are also valid for the completely regular Sturmian system

$$
\begin{gathered}
\ell(y)(x)+\mu r(x) y(x)=\lambda \tau(x) y(x), \quad x \in(0, l), \\
y(x) \in \text { B.C., } \quad \mu \in[0,1] .
\end{gathered}
$$

Lemma 1. The following relation holds:

$$
\mu_{k}-\frac{\mu M}{\tau_{0}} \leq \lambda_{k}(\mu) \leq \mu_{k}+\frac{\mu M}{\tau_{0}},
$$

where $\mu_{k}$ is the kth eigenvalue of problem (1), (2) with $r(x) \equiv 0, \lambda_{k}(\mu)$ is the kth eigenvalue of problem (8), and

$$
\begin{gathered}
\tau_{0}=\min _{x \in[0, l]} \tau(x), \quad M=\max \left\{\left|r_{0}\right|,\left|r_{1}\right|\right\}, \\
r_{0}=\min _{x \in[0, l]} r(x), \quad r_{1}=\max _{x \in[0, l]} r(x) .
\end{gathered}
$$

The proof of Lemma 1 is based on Courant's principle concerning the minimax properties of eigenvalues [5].

Together with problem (1), (2), consider the nonlinear approximation problem

$$
\begin{gathered}
(\ell y)(x)+r(x)\|y(x)\|_{3}^{\varepsilon} y(x)=\lambda \tau(x) y(x), \quad x \in(0, l), \\
y(x) \in \text { B.C. }
\end{gathered}
$$

where $\varepsilon \in(0,1]$.

Note that, for each fixed $\varepsilon \in(0,1]$, we have

$$
r\|y\|_{3}^{\varepsilon} y=o\left(\|y\|_{3}\right) \quad \text { as } \quad\|y\|_{3} \rightarrow 0
$$

therefore, by virtue of Theorem 2.2 from [2], for any $k \in N$ and $v=+$ or - , there exists an unbounded continuum $C_{k, \varepsilon}^{v}$, of solutions to problem (10) such that

$$
\left(\mu_{k}, 0\right) \in C_{k, \varepsilon}^{v} \subset\left(\mathbf{R} \times S_{k}^{v}\right) \cup\left\{\left(\mu_{k}, 0\right)\right\} .
$$

Lemma 2. There exists $a \delta_{0} \in(0,1)$ such that, for any $\varepsilon \in\left(0, \delta_{0}\right)$ and $\gamma_{0}>0$, there exists no nontrivial solution $(\lambda, w)$ of problem (10) satisfying the conditions $w \in S_{k}^{\nu}, k \leq m_{0},\|w\|_{3} \leq \varepsilon$, and $\operatorname{dist}\left(\lambda, I_{0}\right)=\gamma_{0}$, where

$$
I_{0}=\left[\mu_{1}-\frac{M}{\tau_{0}}, \mu_{m_{0}}+\frac{M}{\tau_{0}}\right] \text {. }
$$

Scheme of proof of Lemma 2. Suppose that there exists a sequence $\left\{\left(\xi_{n}, \vartheta_{n}\right)\right\}_{n=1}^{\infty} \subset E$ of solutions of problem (10) corresponding to $\varepsilon_{n}<\frac{1}{n}$, for which

$$
\operatorname{dist}\left(\lambda_{n}, I_{0}\right)=\gamma_{0} \text { and } \vartheta_{n} \in S_{k}^{v}, k \leq m_{0},\left\|\vartheta_{n}\right\|_{3}<\frac{1}{n} \text {. }
$$

Let $w_{n}=\frac{\vartheta_{n}}{\left\|\vartheta_{n}\right\|_{3}}$. Then $w_{n}$ satisfies the relations

DOKLADY MATHEMATICS Vol. $85 \quad$ No. 32012 


$$
\ell\left(w_{n}\right)+r\left\|\vartheta_{n}\right\|_{3}^{\varepsilon_{n}} w_{n}=\xi_{n} \tau w_{n}, \quad w_{n} \in \text { B.C. }
$$

By virtue of (11), the sequence $\left\{\left(\xi_{n}, w_{n}\right)\right\}_{n=1}^{\infty}$ has a subsequence $\left\{\left(\xi_{n_{s}}, w_{n_{s}}\right)\right\}_{s=1}^{\infty}$ converging to $\left(\lambda^{*}, w\right)$ in $E\left(\mathbf{R} \times C^{4}[0, l]\right)$. Moreover, we have $\|w\|_{3}=1, w \in S_{k}^{v}$, $\left\|\vartheta_{n_{s}}\right\|^{\varepsilon_{n_{s}}} \rightarrow \gamma, s \rightarrow \infty, 0 \leq \gamma \leq 1$, and

$$
\begin{gathered}
\ell(w)+\gamma r w=\lambda^{*} \tau w, \\
w \in \text { B.C. }
\end{gathered}
$$

Since $w \in S_{k}^{v}, k \leq m_{0}$, it follows from (12) and (9) that $\lambda^{*} \in I_{0}$. However, this contradicts the inequality $\operatorname{dist}\left(\lambda^{*}, I_{0}\right)=\gamma_{0}>0$, which proves the lemma.

Let $d_{0}$ and $d_{1}$ be the nonnegative numbers determined by

$$
\begin{gathered}
d_{0}=\min _{k=\overline{1, \ldots, m_{0}}}\left\{\mu_{k+1}-\mu_{k} \mid \mu_{k+1} \neq \mu_{k}\right\}, \\
d_{1}=\inf \left\{z \in R_{+} \mid r(x)+z \tau(x)>0, x \in[0, l]\right\} \geq 0 .
\end{gathered}
$$

Theorem 1. The eigenvalues of the completely regular Sturmian system (1), (2) are real and simple, except, possibly, the first $m_{0}$ of them; these eigenvalues form an infinite monotonically increasing sequence $\lambda_{1} \leq \lambda_{2} \leq \ldots \leq$

$\lambda_{m_{0}}<\lambda_{m_{0}+1}<\ldots$, and if $\frac{r_{1}}{\tau_{0}}+d_{1}<d_{0}$, then all eigenvalues are simple. Moreover, each eigenfunction $\vartheta_{k}(x)$, which corresponds to the eigenvalue $\lambda_{k}$, has $k-1$ simple zeros in the interval $(0, l)$ (to be more precise, $\vartheta_{k} \in S_{k}$ ).

Scheme of proof of Theorem 1. Suppose that $k \leq m_{0}$. Since $C_{k, \varepsilon}^{v}$ is a connected set, it follows that, for any $\varepsilon \in\left(0, \delta_{0}\right)$, problem (10) has a solution $\left(\lambda_{\varepsilon}, y_{\varepsilon}\right)$ such that $\lambda_{\varepsilon} \in I_{0}$ and $\left\|y_{\varepsilon}\right\|_{3}=\delta_{0}$. By virtue of Lemma 2, we can find a sequence $\left\{\varepsilon_{n}\right\}_{n=1}^{\infty}$ such that $\varepsilon_{n} \in\left(0, \delta_{0}\right)$, $\lim _{n \rightarrow \infty} \varepsilon_{n}=0$, and the sequence $\left\{\left(\lambda_{\varepsilon_{n}}, y_{\varepsilon_{n}}\right)\right\}_{n=1}^{\infty}$ converges to a solution $\left(\lambda^{*}, y^{*}\right)$ of problem (1), (2), where $\lambda^{*} \in I_{0}$ and $y^{*} \in S_{k}$. Thus, for each $k \in\left\{1,2, \ldots, m_{0}\right\}$, there exists an eigenfunction $y_{k}=y^{*} \in S_{k}$ of problem (1),
(2) corresponding to an eigenvalue $\lambda_{s}, s \in\left\{1,2, \ldots, m_{0}\right\}$. Using (8) and applying the $\mu$-process (see [1]), we pass from the Sturmian system (1), (2) with $r \equiv 0$ to the Sturmian system (1), (2) with $r \neq 0, x \in[0, l]$. Since the eigenvalues move from their initial values, we can be assume that $s=k$.

The change $\xi=\lambda+d_{1}$ transforms system (10) into the equivalent system

$$
\begin{gathered}
\ell(y)+\tilde{\tilde{r} y}=\xi \tau y, \\
y \in \text { B.C. }
\end{gathered}
$$

where $\tilde{\tilde{r}}=r+d_{1} \tau$. Using the system

$$
\begin{gathered}
\ell(y)+\mu \tilde{\tilde{r} y}=\xi \tau y \\
y \in \text { B.C. }
\end{gathered}
$$

and applying the $\mu$-process, we pass from system (1), (2) to system (13). Since the coefficient $r(x, \mu)=\mu \tilde{\tilde{r}}(x)$ increases as the parameter $\mu$ grows from 0 to 1 , it follows that the eigenvalues do not decrease. Note that if $\frac{r_{1}}{\tau_{0}}+d_{1}<d_{0}$, then the eigenvalues $\xi_{1}(\mu), \xi_{2}(\mu), \ldots, \xi_{m_{0}}(\mu)$ do not coincide during the $\mu$-process; therefore, all of them are simple. This completes the proof of Theorem 1.

\section{ACKNOWLEDGMENTS}

The authors are grateful to Academician V.A. Il'in for useful discussions.

\section{REFERENCES}

1. S. N. Janczewsky, Ann. Math. 29 (2), 521-542 (1928).

2. A. P. Makhmudov and Z. S. Aliev, Differ. Uravn. 29 (8), 1330-1339 (1993).

3. Zh. Ben. Amara and A. A. Vladimirov, Fundam. Prikl. Mat. 12 (4), 41-52 (2006).

4. D. O. Banks and G. J. Kurowski, J. Differ. Equations 24, 57-74 (1977).

5. R. Courant and D. Hilbert, Methods of Mathematical Physics (Interscience, New York, 1953; Gostekhizdat, Moscow, 1945), Vol. 1. 